MATHEMATICS OF COMPUTATION

Volume 71, Number 239, Pages 1263-1270

S $0025-5718(01) 01375-8$

Article electronically published on June 22, 2001

\title{
THE IRREDUCIBILITY OF SOME LEVEL 1 HECKE POLYNOMIALS
}

\author{
D. W. FARMER AND K. JAMES
}

\begin{abstract}
Let $T_{p, k}(x)$ be the characteristic polynomial of the Hecke operator $T_{p}$ acting on the space of level 1 cusp forms $S_{k}(1)$. We show that $T_{p, k}(x)$ is irreducible and has full Galois group over $\mathbf{Q}$ for $k \leq 2000$ and $p<2000$, $p$ prime.
\end{abstract}

\section{InTRODUCTION AND STATEMENT OF RESUlTS}

Let $S_{k}(1)$ denote the space of holomorphic cusp forms of even integral weight $k$ for the full modular group $\Gamma(1)=S L_{2}(\mathbb{Z})$. We will denote by $T_{p, k}(x)$ the characteristic polynomial of the action of the Hecke operator $T_{p}$ on $S_{k}(1)$. (For an introductory reference for these terms see Apostol's book [A].)

A conjecture of Maeda asserts that the Hecke algebra of $S_{k}(1)$ over $\mathbb{Q}$ is simple, and that its Galois closure over $\mathbb{Q}$ has Galois group the full symmetric group. There is even some speculation that $T_{p, k}(x)$ is irreducible in $\mathbb{Q}[x]$ and has full Galois group over $\mathbb{Q}$ for every prime $p$. This conjecture is related to the nonvanishing of $L$-functions [KZ], $\mathrm{CF}$, and to constructing base changes to totally real number fields for level 1 eigenforms HM].

There has been some progress toward this conjecture in recent years. For instance, Maeda's conjecture has been checked for $p=2$ and $k \leq 540[\mathrm{~B}]$, [CF], and various other small cases. Also, we know the following density result of [JO].

Theorem. Let $T_{N, q}^{k, \chi}(x)$ denote the characteristic polynomial of the action of the Hecke operator $T_{q}$ on the space $S_{k}(N, \chi)$ of cusp forms of weight $k$, level $N$, and character $\chi$. Let $q$ and $\ell$ be distinct primes not dividing $N$, and let $\mathcal{L}$ denote a prime ideal lying above $\ell$ in $\mathbb{K}_{k, \chi, N}$ (the finite extension of $\mathbb{Q}$ obtained by adjoining all of the Fourier coefficients of the normalized eigenforms of $\left.S_{k}(N, \chi)\right)$. Then

$$
\#\left\{p<X \quad \mid \quad T_{N, p}^{k, \chi}(x) \equiv T_{N, q}^{k, \chi}(x) \quad(\bmod \mathcal{L})\right\} \gg_{N, \chi, k} \frac{X}{\log X} .
$$

In particular, if $T_{N, p}^{k, \chi}(x)$ is irreducible $\bmod 4 \ell$ for some $p$, then the same holds for a positive proportion of primes $p$.

If we specialize to $N=1$, Conrey, Wallace and the first author have obtained a result similar to the last statement of this theorem with the added benefit that they achieve the condition of having full Galois group as well as irreducibility. They also

Received by the editor January 6, 2000 and, in revised form, September 4, 2000.

2000 Mathematics Subject Classification. Primary 11F11.

The research of the first author was supported in part by the American Institute of Mathematics. We thank the referee for many helpful comments. 
obtained a lower bound of $5 / 6$ for the constant in this case. In this paper we report on calculations which establish:

Theorem 1. The Hecke polynomial $T_{p, k}(x)$ is irreducible and has full Galois group over $\mathbb{Q}$ for $k \leq 2000$ and $p<2000$, $p$ prime.

For the remainder of the paper, $p$ and $\ell$ will represent distinct rational primes, and we will abbreviate " $T_{p, k}(x)$ is irreducible and has full Galois group over $\mathbb{Q}$ " by " $T_{p, k}(x)$ satisfies Maeda's conjecture."

Our calculations have two distinct parts. First we show that if $p<2000$, then $T_{n, k}(x)$ satisfying Maeda's conjecture for some $n$ implies Maeda's conjecture for $T_{p, k}(x)$. This is described in Section 2. In Section 3 we describe calculations which show that $T_{2, k}(x)$ satisfies Maeda's conjecture for $k \leq 2000$.

\section{2. $T_{p, k}(x)$ FOR FIXED $k$}

We show

Proposition 1. If $p<2000, p$ prime, and $T_{n, k}(x)$ satisfies Maeda's conjecture for some $n$, then $T_{p, k}(x)$ satisfies Maeda's conjecture.

The proof involves exploiting the fact that if $T_{n, k}(x)$ satisfies Maeda's conjecture, then this puts severe restrictions on every other $T_{m, k}(x)$.

Lemma 1. Suppose $T_{n, k}(x)$ is irreducible and has full Galois group for some $n$. Then for each $m$ either

a) $T_{m, k}(x)$ is irreducible and has full Galois group, or,

b) $T_{m, k}(x)=(x-a)^{d}$ for some $a \in \mathbb{Z}$.

The proof involves considering the action of $G=\operatorname{Gal}\left(\mathbb{K}_{k} / \mathbb{Q}\right)$ on the Hecke basis for $S_{k}(1)$, where $\mathbb{K}_{k}$ is the field generated by the Fourier coefficients of the Hecke basis. Since $G$ acts on both the individual coefficients and the Hecke basis, if one $T_{m, k}(x)$ is irreducible, then all of the eigenforms are in one Galois orbit. And if $T_{m, k}(x)$ also has full Galois group, then there are no intermediate subfields between $\mathbb{K}_{k}$ and $\mathbb{Q}$.

Thus, if $T_{n, k}(x)$ satisfies Maeda's conjecture, then we need only check that $T_{p, k}(x)$ has at least two distinct roots in order to verify Maeda's conjecture for $T_{p, k}(x)$. Our approach is to show that $T_{p, k}(x)$ has at least two distinct roots mod $\ell$ for some $\ell$. The following result from [CFW] verifies this for $5 / 6$ of all primes $p$.

Lemma 2. Suppose $\operatorname{dim}\left(S_{k}(1)\right) \geq 2$. If $p \not \equiv-1,0,1 \bmod 5$, then $T_{p, k}(x)$ has at least two distinct roots $\bmod 5$, and if $p \not \equiv-1,0,1 \bmod 7$, then $T_{p, k}(x)$ has at least two distinct roots $\bmod 7$.

The proof is by inspection of the factorization of $T_{p, k}(x) \bmod 5$ and $\bmod 7$, which is given in $\mathrm{CFW}$.

By Lemmas 1 and 2, we have Proposition 1 for all $p$ except those in four congruence classes mod 35. That is a total of 45 primes $p<2000$. For those primes we must do some explicit calculations. However, the need for calculation can be further reduced by the following, which is part of Lemma 1 of [CFW].

Lemma 3. If $\ell \geq 5$, then $T_{p, k}(x)$ divides $T_{p, k+\ell-1}(x) \bmod \ell$. 
TABle 1. All pairs $p, k$ with $p<2000, p$ prime, such that Proposition 1 cannot be established by a calculation $\bmod 5,7$, or 11

\begin{tabular}{r|l}
\multicolumn{1}{c|}{$p$} & $k$ \\
\hline 251 & 24,34 \\
379 & 24,34 \\
419 & $28,30,38,40,50$ \\
461 & $28,30,38,40,50$ \\
601 & 32 \\
659 & $28,30,38,40,50$ \\
769 & $28,30,38,40,50$ \\
881 & $28,30,38,40,50$ \\
1231 & $28,30,38,40,50$ \\
1429 & $28,30,38,40,50$
\end{tabular}

The proof is to consider the inclusion $S_{k}(1) \subset S_{k+\ell-1}(1) \bmod \ell$, given by multiplication by the level 1 Eisenstein series $E_{\ell-1}(z)$.

Thus, if $T_{p, k}(x)$ has at least two distinct roots $\bmod \ell$, then so does $T_{p, k^{\prime}}(x)$ for all $k^{\prime} \geq k$ with $k^{\prime} \equiv k \bmod (\ell-1)$.

We will let $\ell=11$ for the remainder of this section. For each of the 45 primes $p$ described above, and for each of the five possible values of $k \bmod 10$, we will determine the smallest $k$ such that $T_{p, k}(x)$ has at least two distinct roots mod 11 . The most favorable situation (for our purposes) is when $T_{p, k}(x)$ has at least two distinct roots $\bmod 11$ for $k=24$ and $28 \leq k \leq 36$. By Lemma 3 this would imply that $T_{p, k}(x)$ has at least two distinct roots for all $k$, so conclusion $b$ ) of Lemma 1 never holds for $T_{p, k}(x)$.

It turns out that this "favorable situation" holds for 35 out of the 45 primes $p<2000$ not covered by Lemma 2 . For the remaining 10 cases we find larger values of $k$ for which $T_{p, k}(x)$ has at least two distinct roots $\bmod 11$. This will leave a small number of exceptional cases to check.

We first summarize the results of our calculations, and then describe the method used for the calculations.

Proposition 2. Table 1 shows all pairs $(p, k)$ with $p$ prime, $p<2000$, such that Lemma 2 does not apply, $\operatorname{dim} S_{k}(1) \geq 2$, and $T_{p, k}(x)$ has only one root $\bmod 11$.

Thus, Proposition 1 is established except for the 40 cases given in Table 1. Those remaining cases were checked by explicitly verifying that the polynomial was irreducible. This completes the proof of Proposition 1.

We briefly review the methods required to generate the Hecke polynomials. For details, see [A]. The Hecke operator $T_{p}$ acts on $S_{k}(1)$ by

$$
\left(T_{p} f\right)(z)=p^{k-1} f(p z)+p^{-1} \sum_{b=0}^{p-1} f\left(\frac{z+b}{p}\right) .
$$

In terms of Fourier expansions, if $f(z)=\sum a(n) q^{n}$ and $\left(T_{p} f\right)(z)=\sum b(n) q^{n}$, then it is easy to check that

$$
b(n)= \begin{cases}a(p n) & \text { if } p \nmid n, \\ a(p n)+p^{k-1} a(n / p) & \text { if } p \mid n .\end{cases}
$$


Thus, given a (partial) Fourier expansion of a basis for $S_{k}(1)$, it is straightforward to find the matrix of $T_{p}$ with respect to that basis.

A basis for $S_{k}(1)$ is given by

$$
B_{k}=\left\{\Delta^{a} E_{4}^{b} E_{6}^{c} \mid a \geq 1, b \geq 0, c=0 \text { or } 1,12 a+4 b+6 c=k\right\} .
$$

Here $E_{k}(z)$ is the weight $k$ Eisenstein series, which has Fourier expansion

$$
E_{k}(z)=1-\frac{2 k}{B_{k}} \sum_{n=1}^{\infty} \sigma_{k-1}(n) q^{n},
$$

and $\Delta(z)$ is the weight 12 cusp form given by

$$
\begin{aligned}
\Delta(z) & =\frac{E_{4}(z)^{3}-E_{6}(z)^{2}}{1728} \\
& =\sum_{n=1}^{\infty} \tau(n) q^{n} .
\end{aligned}
$$

Thus, finding a partial Fourier expansion of a basis for $S_{k}(1)$ is straightforward.

For the cases required in this section, the dimension of $S_{k}(1)$ was at most 5 , a total of 2000 Fourier coefficients were required, and the calculation took less than one day using Mathematica on a personal computer. This is a reflection of the fact that we were able to assume that $T_{p, k}(x)$ was irreducible and had full Galois group for some $p$, and this is an extremely strong assumption. The verification that this assumption holds takes a considerable amount of additional work, and this is described in the next section.

\section{3. $T_{2, k}(x)$ FOR LARGE $k$}

Since the size of the coefficients of $T_{2, k}(x)$ explodes as $k$ grows, it is advantageous to work modulo a suitable prime $\ell$. Thus our strategy for verifying that $T_{2, k}(x)$ satisfies Maeda's conjecture is to find appropriate factorizations of $T_{2, k}(x) \bmod \ell$ for various $\ell$ as described in the following lemma from [CF], which is an elaboration of the corresponding lemma of $[\mathrm{B}$.

Lemma 4. Let $f(x) \in \mathbb{Z}[x]$ be a monic polynomial of degree $d$, with splitting field $\mathbb{K} / \mathbb{Q}$. Suppose there are primes $q, r$ and s such that

i) $f \equiv g_{0} g_{1} \cdots g_{j}(\bmod q)$ for distinct irreducible $g_{i} \in \mathbb{F}_{q}[x]$ with $\operatorname{deg}\left(g_{0}\right)=2$ and $\operatorname{deg}\left(g_{i}\right)$ odd for $i \geq 1$.

ii) $f \equiv h_{0} h_{1} \cdots h_{l}(\bmod r)$ for distinct irreducible $h_{i} \in \mathbb{F}_{r}[x]$ with $\operatorname{deg}\left(h_{0}\right)=p$, where $p>d / 2$ is prime.

iii) $f$ is irreducible modulo $s$.

Then $f$ is irreducible and $\operatorname{Gal}(\mathbb{K} / \mathbb{Q})$ is the full symmetric group $S_{d}$.

In the remainder of this section we will first briefly recall some facts about fast multiplication of polynomials modulo a prime $\ell$ using the discrete fast Fourier transform. We then review how the Berlekamp-Massey algorithm can be used to compute a factor of the characteristic polynomial of a matrix. Finally, we will use these algorithms in conjunction with Lemma 4 to obtain an algorithm for verifying Maeda's conjecture for $T_{2, k}(x)$ for $k=542, \ldots, 2000$.

First, we recall (see Proposition III.2.9 in [ $\mathrm{Ko}]$ ) that we only need the first $k / 12$ coefficients in order to distinguish the cuspforms in $S_{k}(1)$. Thus for computational purposes we will treat these cuspforms as polynomials modulo $x^{N}$ for some 
$N>k / 12$. Now if $N$ is a power of 2 , then we will let $\ell$ be a prime which is 1 modulo $2 N$, and let $\omega$ be a primitive $2 N$-th root of unity modulo $\ell$. Recall that the discrete fast Fourier transform (FFT) allows us to evaluate any polynomial $f$ of degree less than $2 N$ simultaneously at all of the $2 N$-th roots of unity modulo $\ell$ in time proportional to $N \log N$. For a detailed account of the fast Fourier transform, we refer the reader to $[\mathrm{Ch}$. If we wish to multiply two polynomials $f$ and $g$ each of degree less than $N$, then we can use FFT to evaluate each of these at all of the $2 N$-th roots of unity. Note that $h=f g$ is the unique polynomial of degree less than $2 N$ with $h\left(\omega^{i}\right)=f\left(\omega^{i}\right) g\left(\omega^{i}\right)$ for $i=1,2, \ldots, 2 N$. Thus, to determine the coefficients of $h$ all we need to do is solve the linear system:

$$
\left(\begin{array}{ccccc}
1 & \omega & \omega^{2} & \ldots & \omega^{2 N-1} \\
1 & \omega^{2} & \omega^{4} & \ldots & \left(\omega^{2}\right)^{2 N-1} \\
& & & \ddots & \\
1 & \omega^{N} & \left(\omega^{N}\right)^{2} & \ldots & \left(\omega^{N}\right)^{2 N-1}
\end{array}\right)\left(\begin{array}{c}
h_{0} \\
h_{1} \\
\vdots \\
h_{2 N-1}
\end{array}\right)=\left(\begin{array}{c}
h(\omega) \\
h\left(\omega^{2}\right) \\
\vdots \\
h\left(\omega^{2 N-1}\right)
\end{array}\right)
$$

Now, we note that the matrix on the left is a Vandermonde and its inverse is

$$
\frac{1}{N}\left(\begin{array}{ccccc}
1 & \omega^{-1} & \omega^{-2} & \ldots & \left(\omega^{-1}\right)^{2 N-1} \\
1 & \omega^{-2} & \omega^{-4} & \ldots & \left(\omega^{-2}\right)^{2 N-1} \\
& & & \ddots & \\
1 & \omega^{-N} & \omega^{-2 N} & \ldots & \left(\omega^{-N}\right)^{2 N-1}
\end{array}\right)
$$

Therefore, in order to determine the coefficients $h_{0}, \ldots h_{2 N-1}$, we simply need to evaluate the polynomial $\sum_{i=0}^{2 N-1} h\left(\omega^{i}\right) x^{i}$ at all of the $2 N$-th roots of unity and we can once again rely on FFT for this. Thus we can multiply any two polynomials each having degree less than $N$ over $\mathbb{F}_{\ell}$ in $O(N \log N)$ time.

Another algorithm which proved useful for our purposes was the BerlekampMassey algorithm (see [M]). This algorithm takes as input a sequence of integers $a_{0}, a_{1}, \ldots, a_{n}$ and gives as its output the coefficients $c_{1}, c_{2}, \ldots, c_{L}$ of the shortest linear recurrence which generates the input sequence, that is the shortest sequence of numbers $c_{1}, c_{2}, \ldots, c_{L}$ such that

$$
a_{j}=-\sum_{i=1}^{L} c_{i} a_{j-i} \quad j=L, L+1, \ldots
$$

It can be shown (see $\left[\mathrm{M}\right.$, Theorem 3]) that if $L \leq n / 2$, then $c_{1}, c_{2}, \ldots, c_{L}$ is the unique minimal length sequence that generates $a_{0}, a_{1}, \ldots, a_{n}$. The running time of this algorithm is $O(n)$.

Now, let $A$ be a nonsingular square matrix of dimension $d$ and let $f(x)=x^{m}+$ $\sum_{m=0}^{d-1} b_{d-m} x^{m}$ be its characteristic polynomial. Following [W], we note that if for any fixed vector $\vec{V}$, we define a sequence of integers $\left\{v_{m}\right\}_{m \in \mathbb{N}}$ by

$$
v_{m}=\left(A^{m} \vec{V}\right) \cdot\left(\begin{array}{c}
1 \\
0 \\
\vdots \\
0
\end{array}\right)
$$


then for all $j \geq d$ we have $f(A) A^{j-d} \vec{V}=0$, which implies

$$
v_{j}=-\sum_{i=1}^{d} b_{i} v_{j-i} .
$$

Thus, our sequence is generated by the linear recurrence with coefficients $b_{1}, \ldots, b_{d}$. Therefore, given the first $2 d$ terms of this sequence, the Berlekamp-Massey algorithm will return the coefficients $c_{1}, \ldots, c_{L}$ of the unique shortest linear recurrence which generates the $v_{i}$ 's. Let $g(x)=x^{L}+\sum_{i=0}^{L-1} c_{L-i} x^{i}$. Since, the coefficients of $f$ and $g$ generate the same sequence and since $g$ is the minimal such polynomial, one can show that $g \mid f$. Thus, given the first $2 d$ terms of the above sequence, the Berlekamp-Massey algorithm will produce for us a factor of the characteristic polynomial of $A$ in time proportional to $d$. Unfortunately, the time needed to produce the first $2 d$ terms of this sequence is proportional to $d^{3}$. Thus we can find a factor of the characteristic polynomial of $A$ in $O\left(d^{3}\right)$ time, which is a bit better than computing the characteristic polynomial in the straightforward manner which is $O\left(d^{4}\right)$. It is noteworthy that in practice it seems that the Berlekamp-Massey algorithm quite often produces the entire characteristic polynomial of our matrix. In any case, the most difficult aspect of our task proved to be producing primes for which $T_{2, k}(X)$ was irreducible and for this it is sufficient to produce any factor of $T_{2, k}(X)$.

The computation for checking that $T_{2, k}(X)$ satisfied Maeda's conjecture for $540 \leq k \leq 2000$ proceeded as follows. We set $N=512$, the first power of 2 greater than $2 \times 2000 / 12$. We need the extra factor of 2 because we wish to distinguish modular forms in $S_{k}(1)$ for $k \leq 2000$ which are in the image of $T_{2}$. Next, we generated a list of primes which were 1 modulo $2 N$ and a list of corresponding primitive $2 N$-th roots of unity modulo those primes for use with FFT. For each of the primes $p$ on our list, we performed the following calculations. For each weight $540 \leq k \leq 2000$, we first generated $N-1$ coefficients of the rational basis forms for $S_{k}(1)$ and then constructed the matrix giving the action of $T_{2}$ on $S_{k}(1)$ with respect to our choice of basis. The Berlekamp-Massey algorithm was then employed to compute a factor of the characteristic polynomial of $T_{2, k}(X)$ modulo $p$. If the entire polynomial was not computed then we discarded it and proceeded to the next weight. If we were able to obtain the entire characteristic polynomial of $T_{2}$, then by analyzing the degrees of $\operatorname{gcd}\left(T_{2, k}(X), x^{p^{i}}-x\right)$ for $i=1,2, \ldots, \operatorname{deg}\left(T_{2, k}(X)\right)$ we attempted to verify the conditions of Lemma 4 for $T_{2, k}(X)$ modulo $p$. We made note of any successes and then moved up to the next weight. We then selected another prime from our list and carried out the above computation again. We repeated this process until the conditions of Lemma 4 were verified for $T_{2, k}(X)$ for all weights $540 \leq k \leq 2000$. Since all computations were carried out modulo $p$, the growth of the coefficients of the basis forms for $S_{k}(1)$ and $T_{2, k}(X)$ became irrelevant. Also, our selection of primes allowed us to use FFT to quickly multiply forms together, which made construction of the basis much faster.

For the sake of brevity we include Table 2, which contains the last 40 weights and the corresponding primes $q, r$ and $s$ as in Lemma 4. All computations were coded in C and performed on Penn State's IBM SP (a 32 processor machine devoted solely to parallel applications) which allowed us to check for the conditions of Lemma 4 modulo 32 primes simultaneously. The computer time necessary for these computations was roughly 12 weeks. 
TABLE 2.

\begin{tabular}{|c|c|c|c|}
\hline$k$ & $q$ & $r$ & $s$ \\
\hline 1922 & 319489 & 974849 & 16465921 \\
1924 & 1720321 & 1720321 & 11591681 \\
1926 & 1130497 & 188417 & 20635649 \\
1928 & 1032193 & 319489 & 37306369 \\
1930 & 1843201 & 286721 & 13344769 \\
1932 & 1990657 & 114689 & 1130497 \\
1934 & 1097729 & 65537 & 3194881 \\
1936 & 1810433 & 1662977 & 14663681 \\
1938 & 3194881 & 163841 & 35880961 \\
1940 & 270337 & 286721 & 3383297 \\
1942 & 417793 & 417793 & 1146881 \\
1944 & 319489 & 65537 & 35045377 \\
1946 & 7667713 & 925697 & 12042241 \\
1948 & 286721 & 737281 & 3022849 \\
1950 & 6684673 & 925697 & 20914177 \\
1952 & 114689 & 417793 & 737281 \\
1954 & 5767169 & 557057 & 1097729 \\
1956 & 1769473 & 286721 & 13631489 \\
1958 & 1769473 & 147457 & 1810433 \\
1960 & 925697 & 925697 & 26214401 \\
1962 & 974849 & 163841 & 20316161 \\
1964 & 557057 & 270337 & 18128897 \\
1966 & 417793 & 147457 & 21594113 \\
1968 & 1196033 & 147457 & 2424833 \\
1970 & 925697 & 925697 & 17440769 \\
1972 & 4866049 & 270337 & 13631489 \\
1974 & 3383297 & 638977 & 1843201 \\
1976 & 2482177 & 417793 & 925697 \\
1978 & 737281 & 319489 & 15228929 \\
1980 & 6725633 & 319489 & 22552577 \\
1982 & 319489 & 786433 & 3604481 \\
1984 & 147457 & 1196033 & 48906241 \\
1986 & 1179649 & 147457 & 22454273 \\
1988 & 2277377 & 417793 & 40058881 \\
1990 & 778241 & 163841 & 17440769 \\
1992 & 778241 & 188417 & 38256641 \\
1994 & 1318913 & 114689 & 33005569 \\
1996 & 4882433 & 1032193 & 26238977 \\
1998 & 4620289 & 286721 & 53370881 \\
2000 & 2424833 & 974849 & 17522689 \\
\hline & & & \\
\hline
\end{tabular}

\section{REFERENCES}

[A] T. Apostol, Modular functions and Dirichlet series in number theory, GTM 41, SpringerVerlag, (1990). MR 90j:11001

[B] K. Buzzard, On the eigenvalues of the Hecke operator $T_{2}$, J. Number Theory 57 (1996), no. 1, 130-132. MR 96m:11033

[Ch] P. Chiu, Transforms, finite fields, and fast multiplication, Math. Mag. 63 (1990), no. 5, 330-336. MR 93c:11113

[Co] H. Cohen, A course in computational algebraic number theory, Springer-Verlag. (1993). MR 94i:11105 
[CF] J.B. Conrey and D.W. Farmer, Hecke operators and the nonvanishing of $L$-functions, Topics in number theory (University Park, PA, 1997), 143-150, Math. Appl., 467, Kluwer Acad. Publ., Dordrecht, 1999. MR 2000f:11055

[CFW] J.B. Conrey, D.W. Farmer, and P.J. Wallace, Factoring Hecke polynomials modulo a prime, Pacific J. Math. 196 (2000), 123-130. CMP 2001:01

[HM] H. Hida and Y. Maeda, Non-abelian base change for totally real fields, Pacific J. Math., special issue (1997), 189-217. MR 99f:11068

[JO] K. James and K. Ono, A note on the irreducibility of Hecke polynomials. J. Number Theory 73 (1998), no. 2, 527-532. MR 2000a:11063

[Ko] N. Koblitz, Introduction to elliptic curves and modular forms, second edition, GTM 97, Springer-Verlag, (1993). MR 94a:11078

[KZ] W. Kohnen and D. Zagier, Values of $L$-series of modular forms at the center of the critical strip, Invent. Math. 64 (1981), no. 2, 175-198. MR 83b:10029

[M] J. L. Massey, Shift-register synthesis and BCH decoding, IEEE Trans. Inform. Theory. vol IT-15 (1969), no. 1, 122-127. MR 39:3887.

[W] D. H. Wiedemann, Solving sparse linear equations over finite fields, IEEE Trans. Inform. Theory. vol IT-32 (1986), no. 1, 54-62. MR 87g:11166

Department of Mathematics, Bucknell University, Lewisburg, Pennsylvania 17837

E-mail address: farmer@bucknell.edu

Department of Mathematical Sciences, Clemson University, Clemson, South CarOLINA 29634-0975

E-mail address: kevja@clemson.edu 